\title{
Plataforma ROTA: Histórico, Desafios e Soluções para Segurança Pública em Cidades Inteligentes
}

\author{
Gustavo A. Carvalho, Pedro P. Barbosa Neto, Nélio Cacho, Eiji Adachi, Frederico Lopes \\ Universidade Federal do Rio Grande do Norte (UFRN) \\ Natal-RN, Brasil \\ gustavo_carvalho@ufrn.edu.br, pedro_paivaneto@hotmail.com, \\ neliocachoddimap.ufrn.br, eijiadachidimd.ufrn.br, fredeimd.ufrn.br
}

\begin{abstract}
Resumo. Em meio ao problema da segurança pública existente no Brasil, esse artigo apresenta o histórico de desenvolvimento e implantação da plataforma ROTA, uma solução de cidades inteligentes criada com o objetivo de operar sobre essa problemática, discorrendo sobre suas aplicações e o propósito de cada uma delas. Os resultados obtidos pela introdução da solução são exibidos e interpretados, expondo os avanços causados pela plataforma. Por fim, são apresentados novos desafios que deverão ser enfrentados pelo ROTA de forma a alcançar o seu funcionamento ideal.
\end{abstract}

\begin{abstract}
Amidst the problem of public safety in Brazil, this paper presents a developement and deployment history of the ROTA Platform, a smart city solution created to address problem, besides discussing its applications and the purpose of each one. The results obtained from the introduction of the solution are shown and explained, thereby exposing advances caused by the platform. The paper also presents new challenges to be handled by ROTA for achieving its ideal behavior.
\end{abstract}

\section{Introdução}

Os últimos anos têm visto um crescimento cada vez maior da população urbana ao redor do mundo. Desde 2009, a quantidade de indivíduos vivendo em zonas urbanas já ultrapassa a população que vive em zonais rurais [UNDESA 2015], e com o tempo esse fenômeno só tende a se intensificar. Estimativas dizem que até 2050 a população urbana terá alcançado o número de 6,4 bilhões de pessoas ao redor do mundo [Herald 2014].

O desenvolvimento acelerado das cidades traz consigo não só benefícios, mas também uma série de problemas relacionados com o crescimento acelerado e nãoplanejado das áreas urbanas. Entre esses problemas, está o do crescimento das taxas de criminalidade. A segurança pública no Brasil é uma questão recorrente quando se trata de áreas a serem melhoradas. De acordo com dados do Atlas da Violência ${ }^{1}$, produzido pelo Instituto de Pesquisa Econômica Aplicada (IPEA) em parceria com o Fórum Brasileiro de Segurança Pública (FBSP), em 2005 a taxa de homicídios no Brasil era de 26,1 para cada 100 mil habitantes, aumentando em 2015 para aproximadamente 28,9. Esses números são aproximadamente quatro vezes superiores à média global. No estado do Rio Grande do Norte, de 2005 à 2015, a taxa de homicídios aumentou de 13,5 para 44,9, uma variação de aproximadamente $+232,0 \%$, a pior registrada dentre todos os estados brasileiros.

\footnotetext{
${ }^{1}$ http://ipea.gov.br/atlasviolencia/
} 
Em meio a essa realidade, a Universidade Federal do Rio Grande do Norte (UFRN) junto à Secretaria da Segurança Pública e da Defesa Social do Estado do Rio Grande do Norte (SESED-RN) apresentam neste artigo o histórico, as soluções apresentadas e os desafios enfrentados durante o desenvolvimento da plataforma ROTA. A plataforma ROTA é uma solução relacionada ao conceito de cidades inteligentes que busca melhorar a segurança pública da cidade de Natal através da integração de diversas fontes de informação e da integração de novas tecnologias na infraestrutura de segurança já existente.

Esse artigo descreve o estado atual da plataforma ROTA e de suas aplicações, além de apresentar os principais resultados alcançados desde a sua implantação. Por fim, são discutidas tanto novas funcionalidades para a plataforma ROTA como também desafios que ainda podem ser enfrentados pela mesma.

\section{Aplicações de Segurança para Cidades Inteligentes}

A problemática da violência e da falta de segurança é uma que ocorre no mundo todo e da qual sempre se buscam novas soluções para tentar resolvê-la. No âmbito de cidades inteligentes, plataformas como INSPEC ${ }^{2}$ T [Leventakis et al. 2016], Polícia Popular ${ }^{2}$ e Emergência $\mathrm{RJ}^{3}$ vêm ganhando notoriedade por permitirem o registro de denúncias e ocorrências diretamente a partir de qualquer smartphone, reduzindo a "carga" das linhas diretas com a polícia, permitindo um maior detalhamento da ocorrência com a inclusão de arquivos multimídia e de informações adicionais como horário e local das ocorrências.

Por mais que as aplicações mencionadas permitam uma forma digitalizada de cadastrar denúncias e ocorrências, os sistemas policiais em si continuam funcionando de forma tradicional. De fato, nenhuma delas ajuda o trabalho policial na gestão de recursos pelo fato de apenas armazenarem os dados relacionados a ocorrências, sem que haja a organização desses dados para assim transmitir informações relevantes que podem ser utilizadas no dia-a-dia das forças policiais.

\section{A Plataforma ROTA}

Neste contexto atual, a partir de uma iniciativa da Universidade Federal do Rio Grande do Norte (UFRN) e da Secretaria de Segurança Pública e da Defesa Social do Rio Grande do Norte (SESED-RN), surge a plataforma ROTA, cujo objetivo é melhorar a segurança pública através de softwares projetados para aprimorar a infra-estrutura de segurança por trás da cidade. A plataforma ROTA fornece tecnologias para coletar, processar, compartilhar, armazenar e analisar uma vasta quantidade de dados vindos de diversas fontes, transformando dados sortidos em informações [Lopes et al. 2016].

A plataforma ROTA tem como principal objetivo auxiliar o gerenciamento das viaturas policiais nas rondas através da cidade. De forma a atender essa necessidade, a ROTA conta com uma série de aplicativos que cumprem as mais diversas necessidades, como: rastreamento das viaturas a partir de localização GPS, gerenciamento e visualização de todas viaturas ativas, análise de áreas com maior risco de ocorrências, e cadastro de novas ocorrências diretamente para a plataforma. Devido ao foco voltado às viaturas da cidade,

\footnotetext{
${ }^{2} \mathrm{http} / / / \mathrm{www}$. policiapopular.com/

${ }^{3}$ https://goo.gl/T4cdic
} 
a maior parte das funcionalidades da ROTA são voltadas para os membros da Polícia Civil. Porém, ainda assim, a população em geral ainda é a principal fonte de denúncia de ocorrências, sendo a principal usuária do aplicativo de cadastro de ocorrências, que é o único aberto ao público.

A ROTA é composta por três camadas de processamento de dados (Figura 1): A Camada de Integração, a Camada Analítica, e a Camada Facade. A camada de Integração é responsável por unificar os dados disponibilizados por diversas fontes de informação da cidade de Natal, como o órgão de trânsito (DETRAN-RN), o Instituto Técnico-Científico de Polícia (ITEP), e a Secretária de Estado da Segurança Pública e Defesa Social (SESED/RN), para criar informações capazes de ser utilizadas pelas outras camadas da plataforma. Notavelmente, essa camada também é a responsável por adequar os dados para serem propriamente processados pela camada analítica, que a partir disso gerará ainda mais informações a partir dos dados já coletados.

Os dados alimentados à camada analítica são processados pelo motor analítico para gerar e correlacionar informações sobre a frequência e a localização das ocorrências, e os padrões das rotas de patrulha. As novas informações adquiridas permitem um visão mais acurada dos padrões de movimentação da cidade, além de permitir um maior grau de planejamento sobre quais áreas necessitam de maior proteção em quais horários. Ainda na camada analítica, a base de dados de objetos móveis HERMES [Pelekis and Theodoridis 2014] foi utilizada para detectar padrões e dados geométricos que auxiliam na obtenção das informações já citadas.

Por fim, a camada Facade provê todas as informações necessárias para o funcionamento dos aplicativos finais da plataforma ROTA. Devido ao grande número de requisições que podem ser necessárias simultaneamente, a camada Facade foi criada seguindo o modelo chamado de Computação em Nuvem. Tal paradigma permite que os diversos recursos necessários sejam disponibilizados aos usuários sob demanda com o mínimo de esforço computacional [Mell and Grance 2011].

Nas seções a seguir, apresentamos em mais detalhes os principais elementos da arquitetura da plataforma ROTA.

\subsection{ROTA Viatura}

Como tradicionalmente toda a comunicação entre as viaturas policiais e a central de operação é realizada por meio da faixa de rádio policial, o repasse de ordens e informações pelo rádio é a única forma de coordenar as viaturas através da cidade. Esse processo envolve um diálogo constante entre a central e cada uma dos veículos policiais, o que o torna lento e inviável quando um grande número de viaturas estão ativas simultaneamente. A ROTA Viatura [Lopes et al. 2016] surge, então, com o objetivo principal de disponibilizar um meio de comunicação alternativo entre a central e as viaturas, permitindo uma maior flexibilidade ao trabalho da polícia militar nas rondas realizadas através da cidade.

O ROTA Viatura propõe uma solução moderna a esse problema de organização informacional. Através de um aplicativo instalado nos tablets Android presentes em cada uma das viaturas da cidade, é possível adquirir uma maior rapidez na transmissão das informações nos dois sentidos entre a viatura policial e o centro de operações. Por exemplo, a central pode enviar todas as informações sobre uma ocorrência instantaneamente 


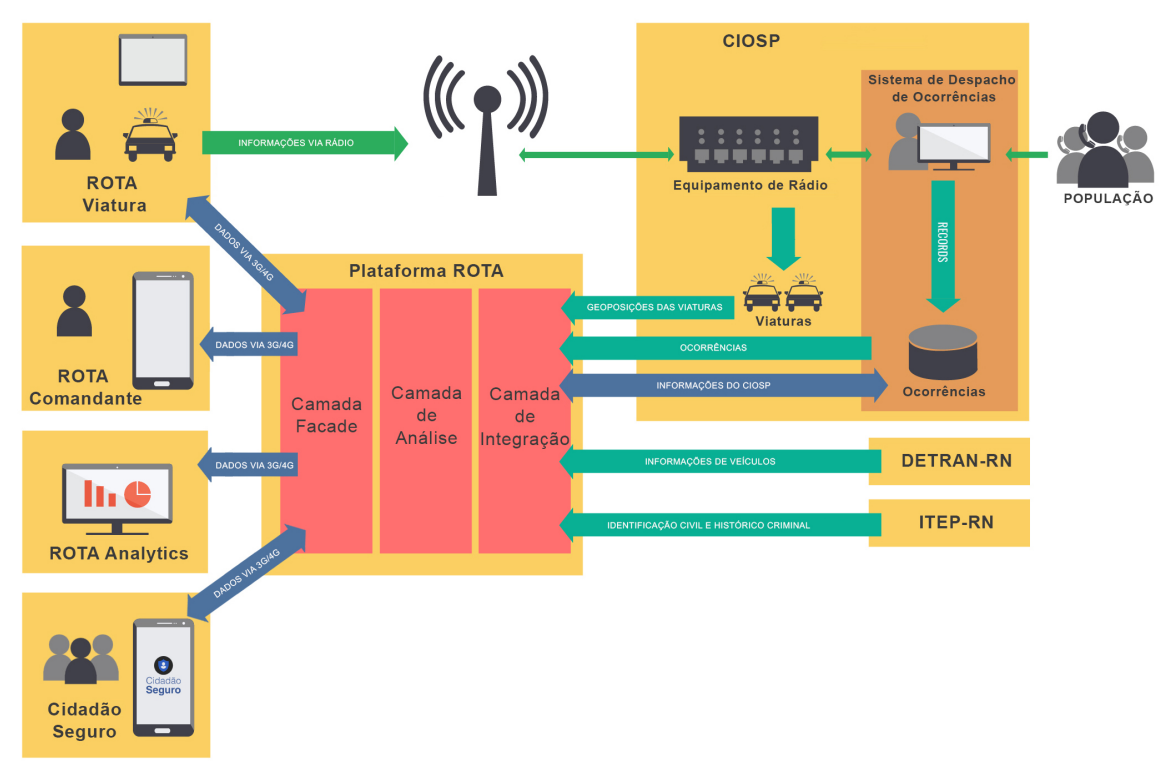

Figura 1. Arquitetura do ROTA

para a viatura policial selecionada, enquanto ao mesmo tempo outra viatura pode requisitar a permissão de reabastecimento, ambos sem a utilização do rádio policial.

Além do mais, a partir dos dados de localização GPS disponibilizados pelos tablets rodando a aplicação, é possível saber com precisão onde cada viatura se encontra em qualquer dado momento. Esses dados permitem um gerenciamento eficaz da frota policial, pois é possível saber a posição e a direção de qualquer veículo sem nem ao menos ser necessário o uso do rádio policial. Isso permite que a central de operações possa gerenciar toda a frota policial de forma ágil, enquanto, ao mesmo tempo, permitindo que o canal de rádio fique livre para transmissões importantes.

\subsection{ROTA Comandante}

Em contraparte ao ROTA Viatura, o ROTA Comandante [Lopes et al. 2016] é o aplicativo da plataforma ROTA que busca facilitar o trabalho da central de monitorar e comandar todas as viaturas circulando pela cidade. $O$ trabalho de determinar a localização de cada viatura, que antes era realizado exclusivamente através da comunicação por rádio, agora passa a ser feito pelo serviço de localização do ROTA Viatura. O ROTA Comandante, que assim como o ROTA Viatura foi criado para especificamente para os tablets Android portados pela polícia, serve como um agregador para essas informações, exibindo os dados de todas as viaturas ativas diretamente sobre o mapa da cidade, tudo em tempo real (Figura 2).

Além disso, o ROTA Comandante também permite a visualização das ocorrências registradas, tanto as que ainda estão em execução quanto as já concluídas recentemente. Com isso, é possível atender as ocorrências ativas rapidamente com as viaturas mais próximas, e ao mesmo tempo também coordenar a patrulha da cidade para cobrir as áreas em que houveram mais ocorrências registradas nos últimos dias.

A partir das informações apresentadas, o comandante pode verificar quais áreas da 


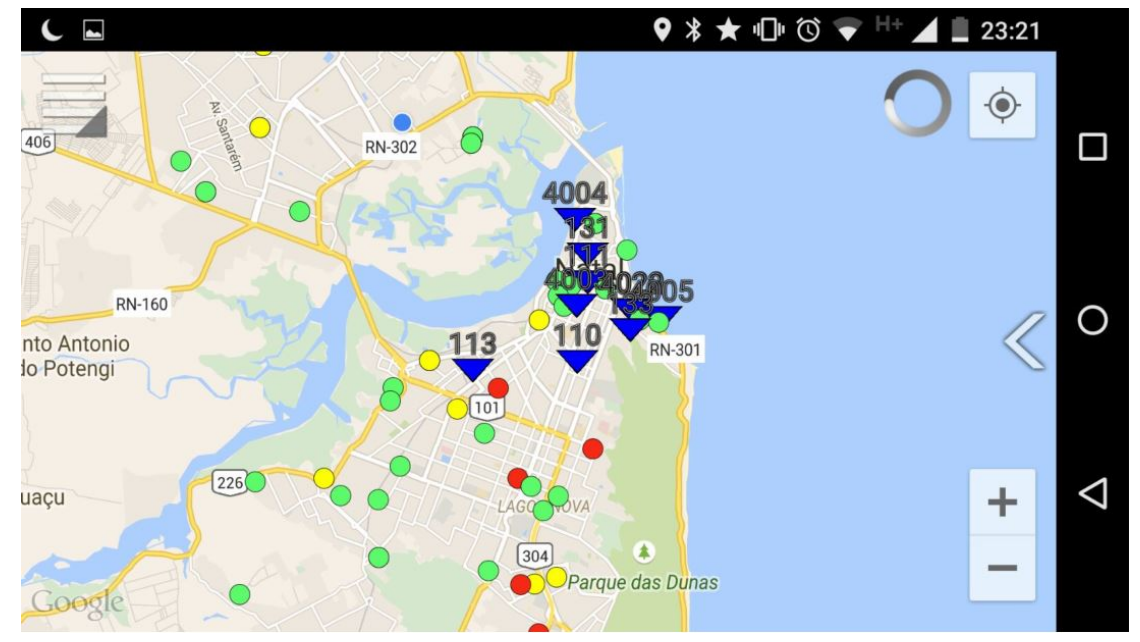

Figura 2. Tela do ROTA Comandante listando viaturas e ocorrências

cidade já estão sendo cobertas e quais ainda carecem de mais viaturas, permitindo, com isso, uma redistribuição do posicionamento das viaturas. Por fim, é possível ainda indicar a rota por qual cada uma delas deve percorrer para satisfazer a sua nova zona de cobertura.

\subsection{ROTA Analytics}

Em cidades inteligentes, no âmbito da segurança, o uso inteligente dos recursos disponíveis é crucial. Nesse contexto, a aplicação ROTA Analytics [Junior 2017] foi criada, e possui como objetivo principal disponibilizar uma previsão da incidência de crimes na cidade.

Essa ferramenta é integrada com o ROTA Viatura, e ajuda o supervisor de patrulha a elaborar uma lista de locais a serem visitados durante a ronda, como também o tempo que deve ser levado em cada um desses lugares. Outra ferramenta importante do ROTA Analytics é mostrar um mapa de calor que subdivide as áreas de atuação dos diferentes distritos de polícia, chamados de Áreas Integradas de Segurança Pública (AISPs), e demonstrar pela diferença de cores entre essas áreas várias métricas específicas, como predição de crimes, densidade de escolas, e outra ferramentas importantes relacionadas à criminalidade (Figura 3).

\subsection{ROTA Cidadão Seguro}

Desde 1995, com a criação do disque-denúncia no Rio de Janeiro, esse era o único método disponível no Brasil para o cidadão entrar em contato com a polícia a respeito de denúncias ou ocorrências. Atualmente, com o aumento vertiginoso tanto da população quanto da parcela que possui acesso a meios de comunicação [OGLOBO 2011], essa ferramenta se encontra sobrecarregada. Nesse contexto, foi criado o aplicativo ROTA Cidadão Seguro [Moreira 2017b] (ou apenas Cidadão Seguro), um projeto que possui como principal proposta o desenvolvimento de um canal de comunicação direta com o Centro Integrado de Operações de Segurança Pública (CIOSP), que "consiste de uma sala de controle responsável por responder chamadas de um número de emergência para os serviços da polícia, bombeiros, e ambulâncias, similar ao 911 nos EUA ou 112 na Europa"[Mendonça 2016]. 


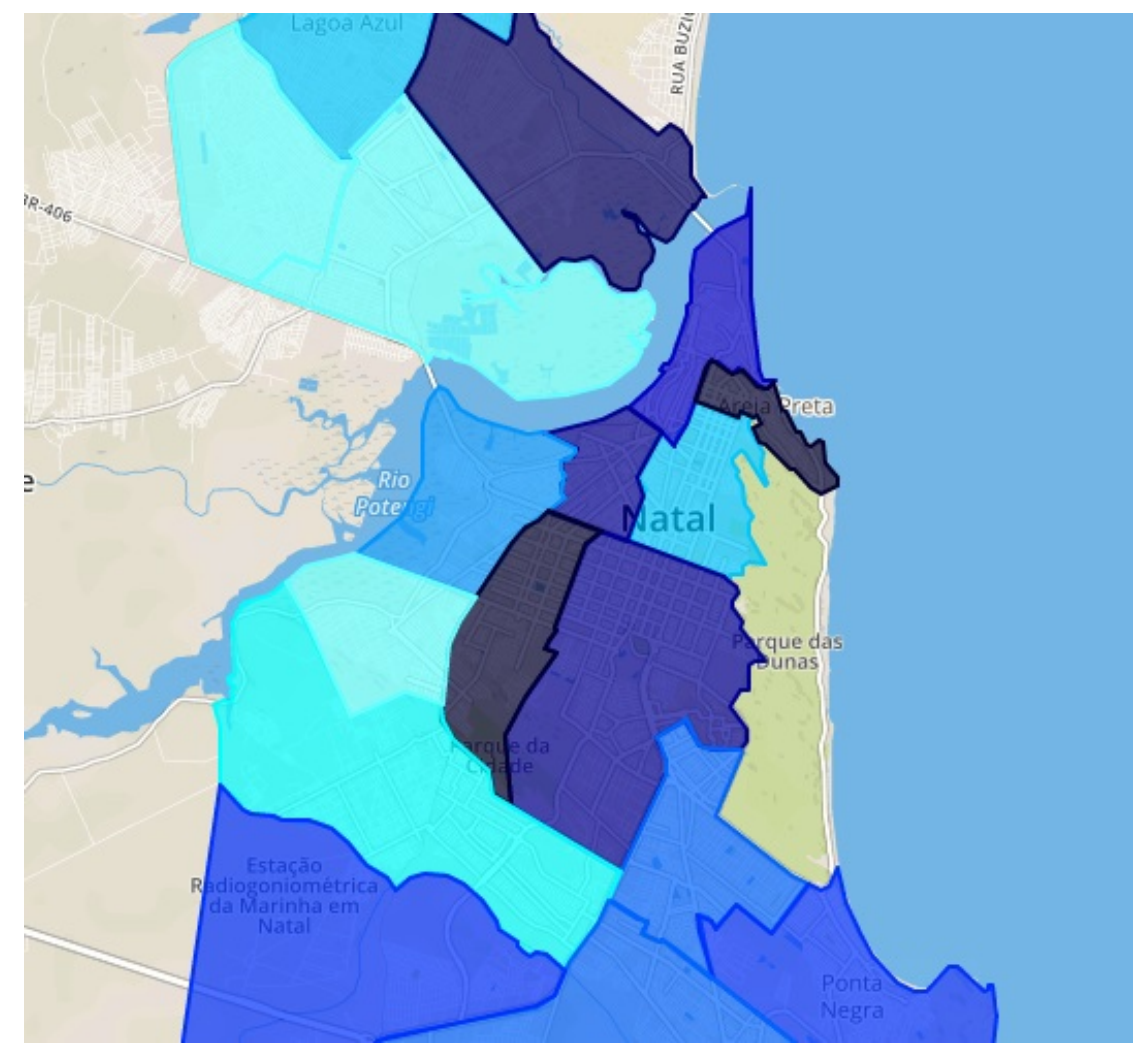

Figura 3. Mapa de Natal dividido pelos departamentos de polícia

Ao utilizar a aplicação, o usuário deverá passar por diferentes módulos para concluir o cadastro de sua ocorrência/denúncia. A partir da criação da ocorrência (Figura 4), devem ser informados dados como tipo de ocorrência, localização, e outras informações relevantes para os policiais que irão averiguá-las, como pontos de referência. Após a criação, o usuário poderá acessar a aplicação para verificar o status de suas ocorrências e assim ter uma forma de feedback, o que não era possível através do disque denúncia. A aplicação está disponível tanto para aplicativos Android quanto iOS, alcançando dessa forma o maior número de usuários possível.

\subsection{Resultados}

A plataforma ROTA trata diversos campos de atuação da segurança pública, além de promover soluções que beneficiam os diferentes atores participantes do processo de criação, gerenciamento, e solução de ocorrências. Esses atores vão desde o cidadão, inserido no processo de registro de denúncias e ocorrências pelo cidadão seguro, aos comandantes, que utilizam os dados analisados pelo Analytics para definir novas rotas para as viaturas utilizando o ROTA Comandante. Essas mudanças afetam diretamente a rotina dos policiais presentes nas viaturas, que podem visualizar informações importantes, como as rotas de patrulha e dados sobre as ocorrências, através do ROTA Viatura. Os benefícios e resultados trazidos por esse processo serão retratados a seguir.

Inicialmente, da aplicação Cidadão Seguro, apesar de ainda não estar disponível para a população em geral, espera-se resultados promissores. A possibilidade de adição de arquivos multimídia, que antes não estava disponível através das linhas telefônicas, 

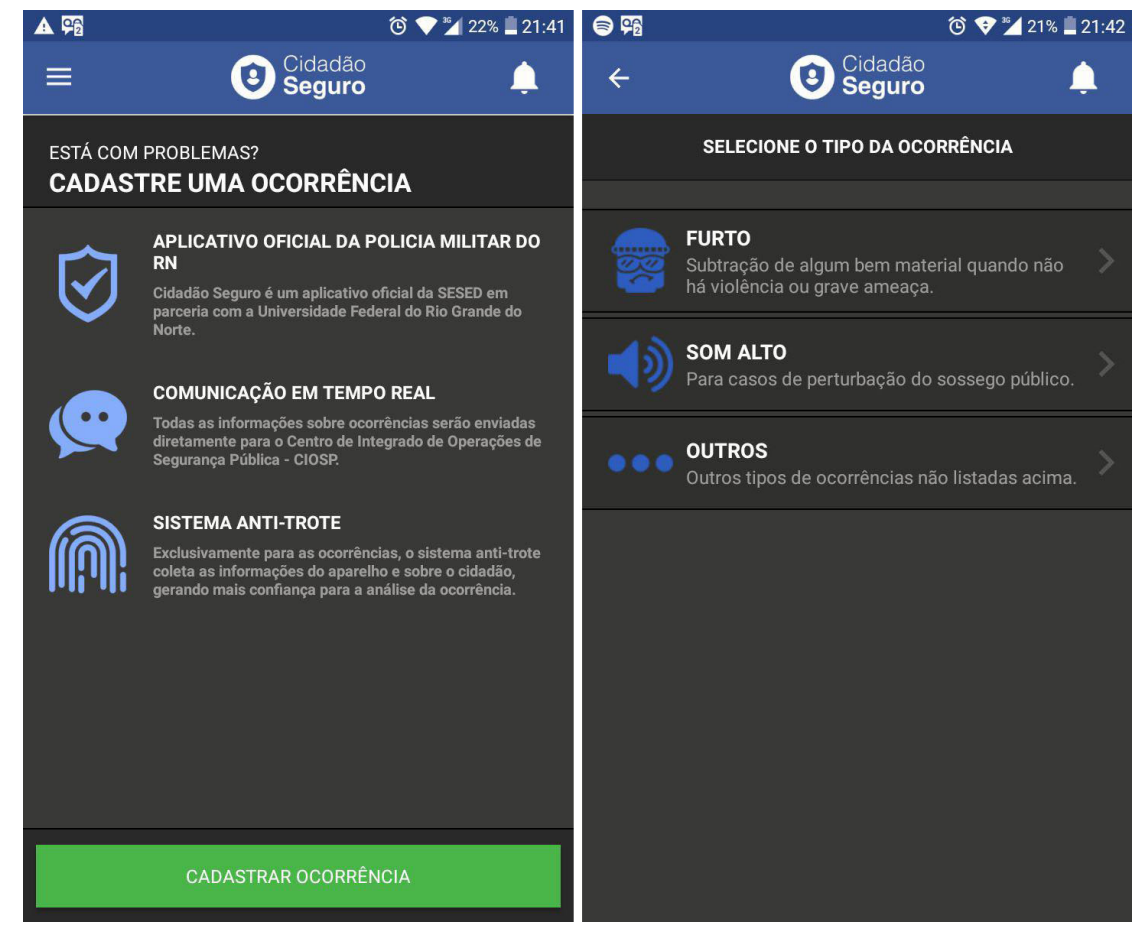

Figura 4. Tela de registro de ocorrências do aplicativo Cidadão Seguro

traz uma riqueza maior de detalhes para os policiais que estarão verificando a denúncia em questão.

Após a criação de novas ocorrências, os policiais militares encarregados de fazer a patrulha e averiguar as mesmas se beneficiam da agilidade e confiabilidade das informações passadas, disponibilizadas pela aplicação do ROTA Viatura. Isso é demonstrado através dos dados coletados desde o início da utilização da aplicação, que foi implantada inicialmente em apenas 19 viaturas, e depois foi expandida pela SESED-RN, chegando a um total de 127 viaturas após um período de 16 meses. Além disso, foram atendidas 1.103 ocorrências, 54.261 buscas de placas e 1.059 buscas de identidades civis nesse mesmo período[Moreira 2017a].

Seguindo o fluxo de resolução das ocorrências, a aplicação ROTA Analytics cumpre o seu objetivo, se tornando uma nova fonte de informações a serem consultadas e que podem ser utilizadas no planejamento de rotas nas viaturas. Assim, otimizando a gestão de recursos policiais nas áreas mais carentes de segurança da cidade.

Com os dados fornecidos pelo ROTA Analytics, em conjuntos com outras ferramentas de análise de dados, agora os comandantes podem, além de gerenciar de forma prática como será efetuada a patrulha, acompanhar a localização delas em tempo real.

De uma forma geral, os resultados obtidos desde a criação da plataforma ROTA mostram que a polícia está aberta a se adaptar para o uso de novas ferramentas que se propõem a agilizar e facilitar o trabalho em suas rotinas.

\section{Novos Desafios}

Por mais que a plataforma ROTA já esteja em operação e tenha diversas das suas aplicações já sendo utilizadas por parte da polícia e dos órgãos de segurança, ainda não 
se pode dizer que as tecnologias estão sendo utilizadas de forma ideal. "Tentativas de implementação de tecnologia muitas vezes falham por causa de questões humanas e organizacionais - em vez de tecnológicas - que foram negligenciadas ou subestimadas no desenvolvimento de soluções de TIC"'[Griffith 1998]. Enquanto as aplicações podem ser compelidas a serem adotadas por parte da polícia, o mesmo não pode ser feito para a população, que só virá a utilizar as aplicações caso confie na sua credibilidade.

Segue como exemplo o aplicativo Cidadão Seguro. O Cidadão Seguro surgiu com o objetivo principal de facilitar as denúncias por parte da população para a polícia e, com isso, passar uma sensação de segurança para todos os usuários do aplicativo. Porém, embora a aplicação promova uma maior facilidade no uso das funcionalidades propostas, não se pode dizer que houve uma melhora significativa no sentimento de segurança do usuário, na sua interação com os órgãos de segurança, ou confiança que o mesmo tem em relação ao trabalho da polícia. "Um dos princípios chave para o policiamento democrático é que a polícia deve operar a partir do melhor interesse da população. Assim sendo, é essencial que a polícia assegure a confiança da população faça o que fizerem”[Emsley 1983].

De forma a contornar essa situação, diversas cidades europeias passaram a utilizar as mídias sociais como forma de aproximar a população da polícia, criando um relação de confiança entre ambos. "As mídias sociais não apenas auxiliam o policiamento comunitário, como também estimulam a introdução do policiamento comunitário. A comunicação autêntica e individual tem um impacto positivo na relação entre um policial e um membro da comunidade e, consequentemente, também no Policiamento Comunitário"[Meijer A 2013]. Ainda segundo [P. Saskia Bayerl and Markarian 2017], "a colaboração da polícia com a comunidade pode evoluir para um policiamento bemsucedido, mais eficaz e eficiente. Isso pode ser alcançado informando a comunidade sobre como ela pode ajudar e explicando-a os limites legais para suas contribuições". Chegando a dizer até que "A mídia social pode ser considerada um elemento básico no policiamento comunitário".

As experiências adquiridas nos projetos europeus podem com isso serem trazidas e integradas também na plataforma ROTA. Um meio de interação descontraído entre a população e a policia, mesmo que realizado fora das redes sociais mais populares, seria uma ferramenta altamente efetiva em aproximar entre ambas as partes, reforçando o policiamento comunitário e facilitando ainda mais o trabalho da polícia. Essa ferramenta teria, além disso, o benefício de reestabelecer a relação de confiança entre a população e a força policial, relação essa que vem comprometida conforme os índices de violência aumentam.

\section{Conclusão}

Esse artigo apresentou um breve histórico sobre a plataforma ROTA, uma solução de cidades inteligentes para o problema da segurança pública na cidade de Natal, apresentando seus aplicativos e a finalidade de cada um deles. Além disso, os resultados obtidos com a implantação dos sistemas foram apresentados e discutidos, dando destaque às mudanças proporcionadas pela ROTA e como a cidade se adequou a elas. Por fim, foi apresentado à plataforma um novo desafio que, apesar de envolver em maior parte somente a população, exerce uma grande influência em como a ROTA será visto pelos habitantes de Natal e em o quão efetivas serão as aplicações que têm como foco principal a população da cidade. 


\section{Referências}

Emsley, C. (1983). Policing and its context, 1750-1870 / Clive Emsley. Macmillan London.

Griffith, T. L. (1998). Cross-cultural and cognitive issues in the implementation of new technology: focus on group support systems and bulgaria. Interacting with Computers, 9(4):431 - 447. Shared Values and Shared Interfaces: The Role of Culture in the Globalisation of Human-Computer Systems.

Herald (2014). City population to reach $6.4 \mathrm{bn}$ by 2050 url: http://www.heraldglobe.com/news/223727231/city-population-to-reach-64bn-by2050, acesso em: 2018-03-31.

Junior, A. A. (2017). A predictive policing application to support patrol planning in smart cities.

Leventakis, G., Papalexandratos, G., Kokkinis, G., Charalambous, E., and Koutras, N. (2016). Towards efficient law enforcement decision support systems in the area of community policing: The use of mobile applications. In 2016 European Intelligence and Security Informatics Conference (EISIC), pages 198-198.

Lopes, F., Coelho, J., Cacho, N., Loyola, E., Tayrony, T., Andrade, T., Medonça, M., Oliveira, M., Estaregue, D., and Moura, B. (2016). Rota: A smart city platform to improve public safety.

Meijer A, T. M. (2013). Social media strategies: understanding the differences between north american police departments.

Mell, P. M. and Grance, T. (2011). Sp 800-145. the nist definition of cloud computing. Technical report, Gaithersburg, MD, United States.

Mendonça, M. (2016). Improving public safety at fingertips: A smart city experience.

Moreira, B. C. (2017a). Plataforma e soluções para segurança pública em cidades inteligentes. TCC (Graduação) - Curso de Ciência da Computação, Departamento de Informática e Matemática Aplicada, Universidade Federal do Rio Grande do Norte, Natal.

Moreira, B. C. (2017b). Towards civic engagement in smart public security.

OGLOBO (2011). Número de celulares no brasil é maior que o de habitantes url: https://oglobo.globo.com/economia/numero-de-celulares-no-brasil-maior-que-dehabitantes-2924116, acesso em 2018-03-31.

P. Saskia Bayerl, Rusa Karlovic, B. A. and Markarian, G. (2017). Community Policing A European Perspective: Strategies, Best Practices and Guidelines. Springer International Publishing.

Pelekis, N. and Theodoridis, Y. (2014). Mobility Data Management and Exploration.

UNDESA (2015). Department of economic and social affairs, population division. world urbanization prospects: The 2014 revision. 